\section{The Importance of Halotherapy in the Treatment of COVID-19 Related Diseases}

\author{
Burhanettin Uysal ${ }^{1^{*}}$, Ebrar Ulusinan ${ }^{1}$
}

${ }^{1}$ Bilecik Seyh Edebali University, Turkey

Correspondence:

Burhanettin Uysal

Address: Bilecik Seyh Edebali University, Turkey

Email: druysal.phd@gmail.com

Received: 13.06 .2020 ,

Accepted: 27.07.2020

https://doi.org/10.29333/jcei/8486

\begin{abstract}
Treatment and post-treatment rehabilitation of COVID-19, which first appeared in Wuhan, China at the end of 2019, causing hundreds of thousands of people to die, has become very important for both patients and scientists. Some chronic health problems, especially in healing patients, have led to the emergence of alternative treatment methods. Significant studies have been conducted on COVID-19 triggering chronic respiratory diseases in patients recovered because of the effects on the respiratory tract. In some studies, it was found that individuals who recovered could not use the functions of the lung with their pre-disease capacity. Halotherapy, which is a treatment method applying by be delivered a fine powder to the upper part of the lower respiratory tract of a person, is a natural and reliable therapy method for respiratory infections, asthma, allergic and chronic bronchitis, colds, pharyngitis, rhinitis, tonsillitis, chronic obstructive pulmonary disease, and various skin diseases. Halotherapy has an important place in alternative medicine. Many studies have been carried out on the benefit of Halotherapy, which is considered both Salt Therapy and Cave Therapy, in chronic respiratory diseases. In this context, it is considered that Halotherapy can be useful in the treatment of chronic respiratory diseases of individuals with COVID-19 disease in our study based on these studies. Also, scientific studies are recommended treating patients with COVID-19 in areas where salt therapy and speleotherapy are involved in the treatment of chronic respiratory diseases.
\end{abstract}

Keywords: COVID-19, halotherapy, respiratory diseases

\section{INTRODUCTION}

Halotherapy (HT), which has no serious side effects, is a natural and reliable therapy method for respiratory infections, asthma, allergic and chronic bronchitis, colds, pharyngitis, rhinitis, tonsillitis, chronic obstructive pulmonary disease (COPD), and various skin diseases. This treatment has become even more popular, especially in recent years $[1,2]$. It is thought that it will include the HT as a definition of alternative medicine, which is a group of therapeutic and diagnostic disciplines outside the institutions where health services are taught and provided [3]. As a matter of fact, it is known that it encompasses body and mental connection based treatments called as "holistic medicine" such as alternative medical science, self-help groups, meditation, yoga or stress management as well as chiropractic, homeopathy, acupuncture, biofeedback, herbal therapy, and massage [4].

The HT is a treatment for a method of transmitting a fine powder to the upper part of a person's lower respiratory tract not only in treatment of common cold and respiratory ailment symptoms because of allergies but also in the provision of general oral and respiratory hygiene [5]. Besides its benefits for these ailments, the HT is preferred for psychosomatic disorders such as stress, fatigue, and headache by creating an environment where people who cannot find a suitable place and leisure time to get rest and fresh air can be freed from the stress of daily life and support their lifestyle $[2,6]$. Although no harm has been revealed in the studies, some side effects such as cough and more abundant secretion can be observed during treatment, but these side effects are actually considered to be a positive effect as 
they contribute to the clearing of the airways [2]. In addition to the drug-free treatment of chronic nonspecific lung diseases [5,7]. The HT is also an effective treatment method that stands out in the treatment of COPD, which is an important health problem in the world $[8,9]$.

Medical care and treatment in COVID-19 outbreaks requiring intensive care treatment, as in previous acute respiratory infections such as SARS (severe acute respiratory syndrome), MERS (Middle East Respiratory Syndrome), avian influenza A (H7N9), and influenza A (H1N1) significantly affect patients' recovery during the outbreak. The most obvious reason for COVID-19 that requires recorded intensive care treatment is acute respiratory distress syndrome (ARDS), which is seen in two-thirds of patients, and respiratory support is extremely important in providing the treatment criteria [10]. The first clinical symptom of COVID-19 that is observed fever and respiratory symptoms in most patients [11] and affects patients over 60 years of age rather than children is pneumonia. And gastrointestinal symptoms and asymptomatic infections are also described [12]. In this context, in a study conducted on 1099 patients in China, it was seen that COVID-19 caused significant damage to the lungs by affecting the respiratory tract, approximately $80 \%$ of patients had pneumonia, and $3.4 \%$ of them had ARDS [13].

In our study, it was aimed to present up-to-date information about the $\mathrm{HT}$, which is used as a treatment method in chronic respiratory and lung diseases caused by COVID-19 and to reveal the literature information.

\section{METHODS}

Our study is theoretical research using the screening method and based on the results of previous studies, to present an alternative treatment method in the treatment of chronic respiratory diseases caused by COVID-19 disease based on the results of the studies. Literature research was performed on the database Google Scholar to research COVID-19 and Halotherapy. As the scanning interval, the option since 2019 was preferred. A total of 4 studies were found in the search using the terms "COVID-19 and Halotherapy", "SARS-CoV-2 and Halotherapy", "Coronavirus Disease 2019 and Halotherapy", "2019-nCov and Halotherapy", "2019 novel coronavirus and Halotherapy". Our study is the first study on the theme of "COVID-19 and Halotherapy". Because both salt therapy and cave therapy are dealt with under the main heading of HT. It is an original study with this aspect.

\section{HALOTHERAPY}

\section{Concept}

The word Halo is briefly referred to as the circle of light (Oxford Learning Dictionary). This word, which is mostly used as a halo effect, is named as an HT in medical studies and it is seen that it is used as an HT in many studies.

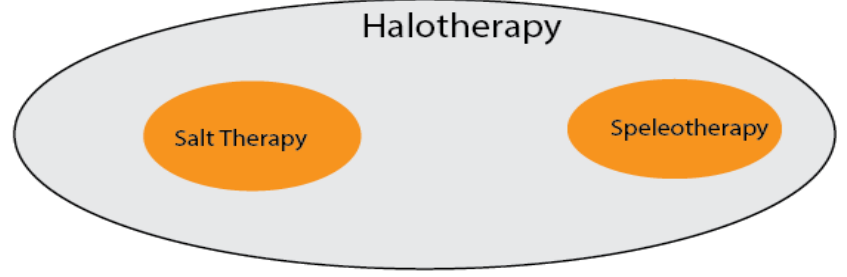

Figure 1. Types of Halotherapy

Vinogradov and Dirksing [5] (1999) defined the HT as the treatment of respiratory diseases by inhaling fine rock salt particles in salt caves or salt mines. The HT, which is a treatment method performed in a special room with saltcovered walls, and also called the halo room [14], is the most accepted practice among modern therapeutic methods based on the application of natural resources and factors [15]. The effectiveness of the HT, which has many health benefits and is used to treat certain diseases, is connected to the unique micro climate in salt caves. The main therapeutic factor in the HT is an atmosphere saturated with dry sodium chloride aerosol with particles from 2 to 5 microns in size. Salt aerosols are formed by the separation and dispersion of fine salt particles from the salt walls [5]. It can be seen that the HT can be used both as a treatment method alone and in combination with some medication and other treatment methods [14]. The treatment process in the HT is important, and in a study conducted by Tibor [16] (1986), it was found that clinical recovery lasted approximately 8 months in the majority of 151 adult patients being treated by the HT.

\section{Types of Halotherapy}

Salt therapy (ST) and speleotherapy, which draw attention with their similarity, will be discussed under two separate headings since there is no clear classification in the literature, it seems more appropriate to evaluate the treatments applied in both the ST and cave therapy as the HT based on the definitions described above. Although it is possible to evaluate the ST under cave therapy, it would be more appropriate to evaluate it separately in terms of the HT treatment.

\section{Salt therapy}

Salt is a substance used by different cultures in many parts of the world due to its health benefits and therapeutic effects [1]. This mineral that is valuable and sacred in some cultures and beliefs, has been widely used in history in many societies and countries in exchange for money, salary, and tax payments. The correct consumption of salt, which must be used for a healthy body, eliminates the risks of disease, and offers a quality life opportunity [17]. There are studies that the consumption of salt as meal salt (sodium chloride) may have benefits or harms in terms of quantity $[18,19]$. However, no meal salt is used in the ST.

The ST was first described in 1843 by Polish therapist Feliks Boczkowski. The fact that the people working in the salt mines in Poland had excellent health despite 
malnutrition and difficult working conditions has been a leading role in the emergence of this treatment. Also, most of the population suffers from colds or respiratory diseases, but the absence of these diseases in people working in salt mines pushed physicians to investigate the salt air that miners breathe every day. Thus, salt mines in Poland and Eastern Europe started to become popular and attract visitors from all over the world. According to studies conducted, salt rooms were found to be three times more sterile than the cleanest operating room [1]. The degree of sterility in the air has a direct and indirect effect on people's health. It is known that chronic respiratory diseases are more common especially in regions with high air pollution.

Especially with the development of industrialization and technology, people are exposed to pollution (air, water, soil, etc.), airborne diseases, allergens, and other harmful factors every day. Salt therapy, which is applied once or twice a week, increases lung capacity, strengthens the immune system, decreases stress, provides better sleep, and revitalizes the whole body because of increased oxygen intake with clearing the lung. The conducted studies show that with the inhalation of dry salt particles, it reduces inflammation in the respiratory tract and expands the airway passages. As dry salt has absorbent properties, it prevents the accumulation of foreign elements that cause some respiratory disorders. A clean respiratory system naturally contributes to higher oxygen uptake, increased energy, and development of the immune system. The ST benefits both athletes and animals as well as adults and children. It is a very beneficial, natural, harmless method that does not have any side effects for the general health condition by improving the function and appearance of the skin and eliminating the rate of poisoning in the respiratory system, strengthening the immune system [20].

\section{Cave therapy (Speleotherapy)}

The ST, which comprises the Latin words 'speleon' (cave) and 'therapium' (treatment) [21], is known as the use of salt mines and cave climate [22,23]. Although the records of the caves used for medical dates back to the Middle Ages, the first scientific studies were carried out in Germany in the 1950s [24]. The principal healing factors of speleotherapy are air quality, subterranean climate, and natural (but higher than normal) radiation levels [25]. The concentration of aerosols of salt caves is $2-5 \mathrm{mg} / \mathrm{m} 3$ diaposone and is especially applied in individuals with respiratory problems [26].

Due to the natural features of the caves, air circulation in the caves is a very important condition. That the caves, where air circulation is not intense and serious, have a clean air away from allergens, which reveals the benefit of not only for tourism but also for therapeutic use in health [27]. Natural salt caves for alleviating the symptoms of chest diseases in Eastern European countries a treatment method because of features such as natural climatic properties, air temperature, moderate and high humidity levels, some polluting factors (sodium, potassium, magnesium, and calcium, etc.), and lack of pollen. This method of therapy is called speleotherapy [8].

Since the humidity and temperature levels in the caves are important in the treatment of disease, in cold caves where the temperature is 6-10 degrees and the relative humidity is $80-100 \%$, it is recommended staying for about an hour a day for three or four weeks and rest in this period. In the caves with a moderate temperature of 13-20 degrees and relative humidity of $45-70 \%$, a longer treatment is carried out 3-4 times a week for about 8-10 hours. In Eastern European countries, a kind of ward is being built in these caves to allow patients to stay longer in these environments. In France, Italy, and Austria, hot caves and old mines with 30-41 degrees and relative humidity of $70-100 \%$ are also used for treatment. Most hot caves have a concentration of radon, an important component of climate therapy. Patients stay in these hot caves for about an hour [25].

Air circulation in the caves is a very important condition and the fact that the caves, where air circulation is not serious, has a clean air away from allergens, not only for tourism but also for treatment in the field of health [27]. In caves and mines used for treatment and health, a cave and a mineral environment such as salt, karst, and potash are chosen first in the treatment process. Then, by drawing a treatment program and the number, duration, and method of application (standing in the environment, exercise, walking, etc.) are determined. The determined treatment is carried out in a certain period by repeating in series, at appropriate intervals specific to the patients and the disease [21].

\section{Halotherapy in Regarding Complementary and Alternative Medicine \\ Complementary medicine is defined by Cochrane Collaboration follows:}

"Complementary and alternative medicine (CAM) is a broad domain of healing resources that encompasses all health systems, modalities, and practices and their accompanying theories and beliefs, other than those intrinsic to the politically dominant health system of a particular society or culture in a given historical period. CAM includes all such practices and ideas self-defined by their users as preventing or treating illness or promoting health and wellbeing. Boundaries within CAM and between the CAM domain and that of the dominant system are not always sharp or fixed" [3].

The use of CAM has increased worldwide over the past decade. Indeed, in the study of Sheikhrabori (2017) and others on 294 diabetic patients, $88.4 \%$ of the participants stated that they used CAM last year [28]. Similarly, in another study, it was concluded that $50 \%$ of the patients $(n=$ 496) used CAM at least once in the past year [29]. 
There are three proposed theories about why alternative medicine is used. These; Dissatisfaction, Need for personal control, and Philosophical congruence. Patients are dissatisfied with medical services because they do not get results from modern medical practices. Low patient satisfaction leads to inefficiency in the services provided. With modern medicine, physicians should focus on machinery and technology in treatment; the technology guiding the treatment instead of the physician, and the high cost of the services provided push the individuals to alternative treatment methods. In this context, alternative medicine is preferred as a very interesting treatment method regarding be close to people's values and worldviews, and being compatible with patients' spiritual and religious values [30].

Looking at WHO Statistics, $88 \%$ of the member states declared that they use complementary medicine. According to 2012 data, acupuncture is the most used practice. Same time, the practice with the most member states reporting availability of health insurance is acupuncture (20 countries in total) [31].

Some of the applications covered by CAM are acupressure, acupuncture (e.g., needle acupuncture, electroacupuncture), aromatherapy, ayurvedic traditional medicine (Ayurveda), Chinese traditional medicine, distant healing, homeopathy, hydrotherapy, hyperbaric oxygenation, hypnosis, massage, meditation, speleotherapy, yoga [32]. As seen the speleotherapy, which is an HT type, is considered to be CAM.

\section{Halotherapy in the Treatment of Diseases}

There are many diseases obtained positive outcomes from the treatment that the HT method is applied. It is known that it is used for therapeutic purposes in colds, which is a heterogeneous disease limited to the upper respiratory [33], in sinusitis, which is an inflammation of the paranasal sinus mucosa [34], in rhinitis, which is defined as inflammation of the membranes covering the nose [35], in pneumonia, which is a serious health problem despite advanced diagnoses and treatments [36], in patients with chronic non-specific pathology of the broncho-pulmonary system and upper respiratory tract, used especially in bronchial asthma, chronic bronchitis [37], in cystic fibrosis, which is characterized by dehydration of airway surface fluid and impaired mucociliary clearance [38], and which is a complex genetic disorder involving the upper respiratory tract [39], in COPD, which is characterized by airflow obstruction $[16,40]$.

The HT is used with $75-85 \%$ efficiency for the treatment and rehabilitation of children with asthma. High efficiency is achieved especially in the treatment of children with recurrent bronchitis. It provides preventive treatment for people with tobacco use and exogenous risk and provides recovery of mucociliary clearance, eliminates early signs of obstruction, and protects the respiratory tract. With a two- week the HT over three months, a 1.5-2 fold reduction in acute viral respiratory infections was achieved in both lung patients and relatively healthy people. Based on its use for vasomotor and allergic rhinitis, it achieved positive results in cases with $72-87 \%$ efficiency [41]. In Turkey and many countries in the world (Romania (Slanic Prahova), France (Solvot's), Russia (Solikamsk), Hungary, countries such as Estonia), there are many caves used for cave therapy. Damlatas cave in Alanya in Turkey is the first cave scientifically studied and found to be beneficial to bronchial asthma and the Çankırı Tuz, Iğdır Tuzluca and Tokat Ballica caves are the most important caves visited for asthma treatment [23].

Causes of Death and Emerging Diseases in COVID-19

\section{Outbreak Process}

In COVID-19, whose pathological features are similar to SARS and MERS [42], respiratory distress caused by ARDS is one of the major causes of death of patients $[43,44]$. Computer Tomography (CT) scans, obtained approximately 10 days after the onset of symptoms on patients recovering from COVID-19, showed that they had a severe lung disease. In chest CT, signs of improvement of the disease began approximately 14 days after the onset of initial symptoms [45]. A case of pneumonia developing due to COVID-19 was diagnosed in a patient detected in Italy on 20 February 2020 [46].

There is quite limited data on the course of development of the disease associated with COVID-19, which does not have a pharmacological treatment and confronts clinicians with great difficulty. It has been concluded that some drugs (corticosteroids) used in previous MERS and SARS outbreaks may also be harmful in COVID-19, and not recommended for use against lung damage and shock therapy [47].

A retrospective study conducted by Ruan et al. [44] (2020) on 150 cases (68 deaths, 82 healed patients) in Wuhan, China, concluded that it not only causes respiratory disorders but also causes heart damage. In a study conducted on 355 patients who died of COVID-19 in Italy, ischemic heart disease was detected in 30\%, diabetes in $35.5 \%$, active cancer in $20.3 \%$, atrial fibrillation in $24.5 \%$, dementia in $6.8 \%$ and stroke in 9.6\% [46].

\section{DISCUSSION AND CONCLUSION}

It is revealed by studies that the demand for alternative medicine is increasing day by day. In a study conducted by MacLennan et al. (1996), it was found that half of the population of South Australia turned to alternative medicines and $1 / 5$ of them offered treatment services to those providing alternative medicine [48]. According to another study conducted by Eisenberg et al. (1998), the use of alternative medicine and spending exploded among 19901997 [49]. In the study conducted by Crocetti et al. (1996), complementary and alternative medical applications were found to be frequently used by patients with cancer [50]. 
Information about prophylaxis and health awareness among people increases as it becomes harder to find a fresh air place. In particular, metropolitan residents are trying to find opportunities to improve their physical and psychological conditions [47]. The HT, an alternative method against numerous respiratory and skin diseases, takes place in a pleasant and relaxed environment that has a positive effect on the psycho-emotional state of the patients [2]. Also, when used in addition to basic medical treatment, the methods developed to increase the effectiveness of the drugs and allow lower doses to be administered. On the other hand, methods tend to eliminate negative responses to the drug and thus increase the effectiveness of the treatment [51].

In a study conducted by Chervinskaya and Zilber [13] (1995), which evaluated the HT effects in 124 patients with various respiratory diseases, $70-80 \%$ (according to nosology) improvement was found after 3-5 sessions of the HT. Patients observed better sputum removal, less cough attacks, and less respiratory disturbances, but also improved sleep, fatigue and weakness, and improved nervous systems. In the overwhelming majority of cases, the number and intensity of asthma attacks and respiratory distress have decreased or disappeared. Similarly, in a pilot study on asthma patients, the halogenated salt chamber appears to provide some beneficial effects in children with mild asthma [52]. Proper monitoring and control of asthmatic patients caught with COVID-19 will slow the triggering of asthma and help control infection [53]. In this respect, it is thought that HTassisted control and follow-up of asthma patients caught and recovering to COVID-19 may be beneficial.

In another study on 12 medium distance runners, after the HT treatment, an increase was observed in the respiratory volumes of all subjects and the percentage of subjects with excellent respiratory index, as well as improvement in breathing, as well as oxygen saturation of arterial blood and resistance to apnea and hypoxia [54].

In a pilot study in which cystic fibrosis (CF) patients took nine sessions of the HT comprising 45 minutes in 3 weeks, it was seen that some benefits may be such as improvement in SNOT-20, Borg Dyspnea scale, physical area, health perception area and digestive area in CF patients with symptomatic sinus disease [38].

In a study of 67 COPD patients recommending the HT to alleviate respiratory discomfort in COPD patients, walking distance and FEV1 improved in patients treated with both the HT and saline breathing, and quality of life (SGRQ) improved in patients treated with the HT [55]. In another study following the course of treatment of COPD patients with the HT, positive symptoms such as reduced cough frequency and intensity, and easier sputum removal were observed in the majority of participants [47].

There have been significant studies that the HT, both as the ST and cave therapy, may be useful in respiratory diseases. When the studies are examined, it is thought that HT may be useful in the treatment of diseases that occur in the COVID-19 process. Scientific studies for the treatment of COVID-19 patients are recommended in areas where the ST and speleotherapy are actively carried out.

Declaration of interest: The authors report no conflicts of interest.

Financial Disclosure: No financial support was received.

\section{REFERENCES}

1. Vladeva E. Halotherapy - An alternative method for the treatment of respiratory diseases. Medical University of Varna, 2015:21(1-2): 31-5. doi: 10.14748/hl.v21i12.5050

2. Vladeva E, Panajotova L. Halotherapy - Benefits and risks. Scripta Scientifica Salutis Publicae, 2018; 4: 22-6. doi: 10.14748/sssp.v4i0.5010.

3. Zollman C, Vickers A. What is complementary medicine?. BMJ. 1999:319(7211):693-6. doi: 10.1136/bmj.319.7211.693.

4. Spiegel D, Stroud P, Fyfe A. Complementary medicine. West I Med 1998:168(4):241-7.

5. Vinogradov V, Dirksing RS. Method of delivering halotherapy. United States Patent Number: 5,881,720. 1999.

6. Lazarescu H, Simionca I, Hoteteu M, Munteanu A, Rizea I, Iliuta A, Dumitrascu D, Dumitrescu E. Surveys on therapeutic effects of "halotherapy chamber with artificial salt-mine environment" on patients with certain chronic allergenic respiratory pathologies and infectious-inflammatory pathologies. Journal of Medicine and Life 2014;7(Spec Iss 2):83-7.

7. Rashleigh R, Smith SM, Roberts NJ. A review of halotherapy for chronic obstructive pulmonary disease. International Journal of Chronic Obstructive Pulmonary Disease 2014;9:239-46. doi: 10.2147/COPD.S57511.

8. Eslaminejad A, Taghavi K, Zohal M, Kialashaki M, Fakharian A. Speleotherapy as an effective treatment of chronic obstructive pulmonary disease. J Respir Med Lung Dis. 2017;2(5):1029. ISSN: 2475-5761.

9. Murthy S, Gomersall CD, Fowler RA. Care for critically Ill patients with COVID-19. JAMA. 2020; 323(15):14991500. doi: 10.1001/jama.2020.3633.

10. Jiang F, Deng L, Zhang L, Cai Y, Cheung CW, Xia Z. Review of the clinical characteristics of coronavirus disease 2019 (COVID-19). J Gen Intern Med. 2020;35(5):1545-9. doi: 10.1007/s11606-020-05762-w.

11. Velavan TP, Meyer CG, The COVID-19 epidemic. Tropical Medicine and International Health 2020;25(5):278-80. doi: 10.1111/tmi.13383.

12. Guan W, Ni Z, Yu H, Liang W, Ou C, He J, et al. Clinical characteristics of 2019 novel coronavirus infection in China. medRxiv preprint posted online on Feb. 9, 2020. doi: $10.1101 / 2020.02 .06 .20020974$. 
13. Chervinskaya AV, Zilber NA. Halotherapy for treatment of respiratory diseases. Journal of Aerosol Medicine 1995;8(3):221-32. doi: 10.1089/jam.1995.8.221.

14. Chervinskaya AV. Prospects of halotherapy in sanatorium-and-spa dermatology and cosmetology. Resort Bulletin 2006;3(36):74-5.

15. Tibor H. Speleotherapy: a special kind of climatotherapy, its role in respiratory rehabilitation. International Rehabilitation Medicine 1986;8:90-2. doi: $10.3109 / 03790798609166185$.

16. Demirkol Ş, Çiftçi İ, Çiftçi H. The importance of rock salt in terms of gastronomy and faith: hacibektaş rock salt, 1 st International Congress of New Generation and New Trends in Tourısm; 2008 November 01-03; Sapanca, Turkey; 2008:298-309.

17. Grassi G, Dell'Oro R, Seravalle G, Foglia G, Trevano FQ, Mancia G. Short- and long-term neuroadrenergic effects of moderate dietary sodium restriction in essential hypertension. Circulation 2002;106:1957-61. doi: 10.1161/01.CIR.0000033519.45615.C7.

18. Cohen HW, Hailpern SM, Fang J, Alderman MH. Sodium intake and mortality in the NHANES II followup study. The American Journal of Medicine 2006;119: 275.e7-275.e14. doi: 10.1016/j.amjmed.2005.10.042.

19. Salt Therapy Association. Benefits of salt therapy. 2018. Available at: https://www.salttherapyassociation.org/ (Accessed: 12 May 2020).

20. Karagülle MZ, Karagülle M. Speleotherapy / Cave treatment; example of tuzluca. The First International Salt Therapy Workshop 2017 3-4 July; Iğdır, Turkey, 2017:37-8.

21. Munteanu C, Hoteteu M, Munteanu D. Therapeutic properties of salt mines From Romania. The First International Salt Therapy Workshop; 2017 3-4 July; Iğdır, Turkey, 2017: 14-21.

22. Demir B. Therapeutic geology (The therapeutic effects of geological materials, geological processes and geological place). Journal of Geological Engineering 2009;33(1):63-78. Available at https://dergipark.org.tr/ tr/pub/jmd/issue/52383/686281

23. Katalin N, Norbert K, Tibor K, Janos S. Radon therapy and speleotherapy in Hungary. La Presse Thermale Et Climatique, 2008;145:219-25.

24. Beamon SP, Falkenbach A, Fainburg G, Linde K. Speleotherapy for asthma. Cochrane Database of Systematic Reviews 2001;2:1-10. doi: 10.1002/14651858.CD001741.

25. Gül Ü. Treatment of existence of salt particle environment in skin diseases and cosmetology: Speleotherapy and Halotherapy, The First International Salt Therapy Workshop; 2017 3-4 July; Iğdır, Turkey, 2017: 39-41.

26. Zeybek Hİ, Aylar F, Dinçer H. Kılıçlı Cave (Kastamonu) and its importance for tourism. International Journal of Geography and Geography Education, 2019;40:363-81.
27. Heikkinen T, Järvinen A. The common cold. The Lancet 2003;361:51-9.

28. Sheikhrabori A, Dehghan M, Ghaedi F, Khademi GR. Complementary and alternative medicine usage and its determinant factors among diabetic patients: An Iranian case. Journal of Evidence-Based Complementary \& Alternative Medicine, 2017:22(3):449-54. doi: $10.1177 / 2156587216675079$.

29. Hori S, Mihaylov I, Vasconcelos JC, et al. Patterns of complementary and alternative medicine use amongst outpatients in Tokyo, Japan. BMC Complement Altern Med 2008;8:14. doi: 10.1186/1472-6882-8-14.

30. Astin JA. Why Patients Use Alternative Medicine: Results of a National Study. JAMA. 1998;279(19):154853. doi: 10.1001/jama.279.19.1548.

31. World Health Organization, 2019. WHO Global Report on Traditional And Complementary Medicine 2019.

32. Wieland LS, Manheimer E, Berman BM. Development and classification of an operational definition of complementary and alternative medicine for the Cochrane collaboration. Altern Ther Health Med. 2011;17(2):50-9.

33. Shapiro GG, Rachelefsky DS. Introduction and definition of sinusitis. Journal of Allergy and Clinical 1992;90(3):417-8. doi: 10.1016/0091-6749(92)90160-4.

34. Dykewicz MS, Fineman S, Skoner DP, Nicklas R, Lee R, Blessing Moore J, et al. Diagnosis and management of rhinitis: complete guidelines of the joint task force on practice parameters in allergy, asthma and immunology. american academy of allergy, asthma, and immunology. Ann Allergy Asthma Immunol 1998;81:478-518. doi: 10.1016/S1081-1206(10)63155-9.

35. Eser İ, Günay Ş, Sak ZHA, Yalçın F, Kürkçüoğlu İC. Mean platelet volume as a diagnostic indicator in pneumonia disease. Tıp Araştırmaları Dergisi 2014;12(1):12-4. doi: 10.18827/etad.83923.

36. Lemko OI, Lemko IS. (2017). Speleotherapy, halotherapy, haloaerosoltherapy: definitions, mechanisms of influence, perspectives of usage (part I). Астма Та Алергія 2017; 2:1-10 ISSN 2307-3373.

37. Flume PA, Robinson KA, O'Sullivan BP, Finder JD, Vender RL, Willey-Courand DB, White TB, Marshall BC. Cystic fibrosis pulmonary guidelines: airway clearance therapies. Respir Care 2009;54(4):522-37.

38. Achkar MA, Geller DE, Slaney AP, Layish DT. Halotherapy in patients with cystic fibrosis: a pilot study. International Journal of Respiratory and Pulmonary Medicine 2015;2(1):1-3. doi: 10.23937/2378$3516 / 1410009$.

39. National Institute for Health and Care Excellence, Health and Social Care Directorate Quality standards Process guide Access date: 02 March 2020. Available at: www.nice.org.uk2015

40. Chervinskaya AV. Available at: https://chervinskaya. com/ (Accessed: 12 May 2020). 
41. Xi Z, Shi L, Wang Y, Zhang J, Huang L, Zhang C et al. Pathological findings of COVID-19 associated with acute respiratory distress syndrome. Lancet Respir Med 2020;8:420-22. doi: 10.1016/S2213-2600(20)30076-X.

42. Mehta P, McAuley DF, Brown M, Sanchez E, Tattersall RS, Manson JJ. COVID-19: consider cytokine storm syndromes and immunosuppression. The Lancet 2020;395:1033-4. doi: 10.1016/S0140-6736(20)30628-0.

43. Ruan Q, Yang K, Wang W, Jiang L, Song J. Clinical predictors of mortality due to COVID-19 based on an analysis of data of 150 patients from Wuhan, China. Intensive Care Med 2020. doi: 10.1007/s00134-02005991-x

44. Pan F, Ye T, Sun P, Gui S, Liang B, Li L, et al. Time course of lung changes at chest ct during recovery from coronavirus disease 2019 (COVID-19). Radiology 2020;295(3):715-21. doi: 10.1148/radiol.2020200370.

45. Onder G, Rezza G, Brusaferro S. Case-Fatality rate and characteristics of patients dying in relation to COVID19 in Italy. JAMA. 2020;323(18):1775-6. doi: 10.1001/jama.2020.4683.

46. Russell CD, Millar JE, Baillie JK. Clinical evidence does not support corticosteroid treatment for 2019-nCoV lung injury. The Lancet 2020;395:473-5. doi: 10.1016/S0140-6736(20)30317-2.

47. Zając J, Bojar I, Helbin J, Kolarzyk E, Owoc A. Salt caves as simulation of natural environment and significance of halotherapy. Annals of Agricultural and Environmental Medicine 2014;21(1):124-7.

48. MacLennan AH, Wilson DH, Taylor AW. Prevalence and cost of alternative medicine in Australia. Lancet. 1996;347(9001):569-73. doi: 10.1016/s01406736(96)91271-4.
49. Eisenberg DM, Davis RB, Ettner SL, et al. Trends in alternative medicine use in the United States, 1990-1997: results of a follow-up national survey. JAMA. 1998;280(18):1569-75. doi: 10.1001/jama.280.18.1569.

50. Crocetti E, Crotti N, Montella M, Musso M. Complementary Medicine and Oncologists' Attitudes: A Survey in Italy. Tumori. 1996;82:539-42. doi: 10.1177/030089169608200605.

51. Fainburg GZ. (2017). Speleotherapy in potash mines and speleoclimatotherapy in sylvinite speleorooms as important directions of salt therapy, The First International Salt Therapy Workshop; 2017 3-4 July; Iğdır, Turkey, 2017. p. 1-7.

52. Bar-Yoseph R, Kugelman N, Livnat G, Gur M, Hakim F, Nir V, Bentur L. Halotherapy as asthma treatment in children: A randomized, controlled, prospective pilot study. Pediatric Pulmonology 2017;52:580-7. doi: 10.1002/ppul.23621.

53. Shaker MS, Oppenheimer J Grayson M, Stukus D, Hartog D, Hsieh EWY, et al. COVID-19: Pandemic contingency planning for the allergy and immunology clinic. The Journal of Allergy and Clinical Immunology: In Practice 2020;8(5):1477-1488.e5, ISSN 2213-2198, doi: 10.1016/j.jaip.2020.03.012.

54. Catalina S, Catalin S, Ion S. Impact assessment of saline aerosols on exercise capacity of athletes. Procedia Social and Behavioral Sciences 2012;46:4141-5. doi: 10.1016/j.sbspro.2012.06.214.

55. Weinreich UM, Nilsson T, Mylund L, Christiansen HT, Laursen BS. Salt halo therapy and saline inhalation administered to patients with chronic obstructive pulmonary disease: A pilot study. Journal of Palliative Care \& Medicine 2014;4(4):1-5. doi: 10.4172/21657386.1000185 . 